\title{
From Computational to Human Trust: Problems, Methods and Applications of Trust Management
}

\author{
Adam Wierzbicki \\ Polish-Japanese Institute of Information Technology (PJIIT) \\ Ul. Koszykowa 86, 02-008 Warsaw, Poland \\ adamw@pjwstk.edu.pl
}

\begin{abstract}
The tutorial will be devoted to trust management mechanisms and their practical applications, such as reputation systems for online auctions and recommendation systems.
\end{abstract}

\section{Introduction}

The tutorial will be devoted to trust management mechanisms and their practical applications, such as reputation systems for online auctions (like eBay) and recommendation systems (like epinions).

The first aim of the tutorial will be to familiarize participants with trust management methods and to give a background for researchers interested in this subject. The tutorial will also demonstrate various research methods used in the area, from the analysis of large datasets to surveys and experimental games. The tutorial will include a specially designed experimental game that will allow participants to get a feeling for the more abstract concepts used in trust management, as well as to understand the basic issues of trust management system design.

The second goal of the tutorial is to thoroughly cover two areas of trust management: reputation systems for online auctions and trust propagation algorithms for recommendation systems. These areas will be discussed in detail, covering relevant research results and the author's own work. The tutorial will conclude by a discussion of possible research directions in the area of trust management. 